\title{
Combined effects of concurrent Pc5 and chorus waves on relativistic electron dynamics
}

\author{
C. Katsavrias ${ }^{1,2}$, I. A. Daglis ${ }^{1}$, W. Li $^{3}$, S. Dimitrakoudis ${ }^{4}$, M. Georgiou ${ }^{1}$, D. L. Turner ${ }^{5}$, and C. Papadimitriou ${ }^{1}$ \\ ${ }^{1}$ Department of Physics, National and Kapodistrian University of Athens, Athens, Greece \\ ${ }^{2}$ Department of Physics, Aristotle University of Thessaloniki, Thessaloniki, Greece \\ ${ }^{3}$ Department of Atmospheric and Oceanic Sciences, UCLA, California, USA \\ ${ }^{4}$ Institute for Astronomy, Astrophysics, Space Applications \& Remote Sensing, \\ National Observatory of Athens, Penteli, Greece \\ ${ }^{5}$ The Aerospace Corporation, El Segundo, California, USA \\ Correspondence to: C. Katsavrias (ckatsavrias@phys.uoa.gr)
}

Received: 9 June 2015 - Revised: 26 August 2015 - Accepted: 12 September 2015 - Published: 25 September 2015

\begin{abstract}
We present electron phase space density (PSD) calculations as well as concurrent Pc5 and chorus wave activity observations during two intense geomagnetic storms caused by interplanetary coronal mass ejections (ICMEs) resulting in contradicting net effect. We show that, during the 17 March 2013 storm, the coincident observation of chorus and relativistic electron enhancements suggests that the prolonged chorus wave activity seems to be responsible for the enhancement of the electron population in the outer radiation belt even in the presence of pronounced outward diffusion. On the other hand, the significant depletion of electrons, during the 12 September 2014 storm, coincides with long-lasting outward diffusion driven by the continuous enhanced Pc5 activity since chorus wave activity was limited both in space and time.
\end{abstract}

Keywords. Magnetospheric physics (energetic particles trapped)

\section{Introduction}

The relativistic electron population in the outer radiation belt is extremely variable - especially during periods of enhanced geomagnetic activity. During such times, it is continually subjected to loss and acceleration processes, which compete and can deplete, enhance or cause little (or no) effect on the electron population (Reeves et al., 2003; Turner et al., 2013).

The mechanisms responsible for the acceleration or loss can be generally divided into two categories: adiabatic or re- versible changes (e.g., $D_{\mathrm{ST}}$ effect) and non-adiabatic or irreversible changes.

Concerning the non-adiabatic relativistic electron acceleration two mechanisms act (usually) together: (i) inward radial diffusion and (ii) local acceleration via wave-particle interactions through whistler mode chorus waves. For inward radial diffusion, electrons in the plasma sheet represent a source of high phase space density (PSD), and when the third adiabatic invariant is broken these particles can diffuse inwards in radial distance, gaining energy in the process (Schulz and Lanzerotti, 1974). For in situ acceleration by wave-particle interactions, substorm injections and enhanced convection are responsible for providing a seed population of tens to a few hundreds $\mathrm{keV}$ electrons which can then be accelerated to higher energies, of the order of $\approx \mathrm{MeV}$, as they interact with lower band chorus waves inside the outer belt (Horne et al., 2005). Some of these injected particles can also generate plasma waves responsible for gyro-resonant acceleration of electrons to relativistic energies (O'Brien et al., 2003; Baker and Daglis, 2007; Bortnik et al., 2007). As shown by Meredith et al. (2001) chorus emissions are often substorm dependent and chorus emissions are generally enhanced when substorm activity is enhanced. Moreover, the equatorial chorus is strongest in the lower band during active conditions in the region $3<L<7$, between 23:00 and 13:00 MLT, consistent with keV electron injection from substorms near midnight and subsequent drift around dawn to the dayside. 
Non-adiabatic (true) losses, on the other hand, are believed to be dominated by two mechanisms: (i) rapid scattering into the atmospheric loss cones (drift or bounce) via wave-particle interactions due to plasmaspheric hiss, electromagnetic ion cyclotron (EMIC) or chorus waves (Shprits et al., 2007) and (ii) magnetopause shadowing combined with outward radial transport (Turner et al., 2012; Shprits et al., 2006). Recently, Usanova et al. (2014) combined electron precipitation observations and simulations to show that EMIC waves were able to affect the low pitch angle electrons in the outer belt, but not the core of the electron distribution during the 11 October 2012 event. In addition, Jaynes et al. (2014) showed that in the absence of additional energization, plasmaspheric hiss was responsible for continuous losses of electrons inside the plasmasphere during the time period 22 December 2012 through 13 January 2013.

Turner et al. (2012) showed that the majority of nonadiabatic losses of outer radiation belt electrons with energies above $300 \mathrm{keV}$ during the main phase of the 6 January 2011 geomagnetic storm were not lost to the atmosphere but to Earth's magnetopause through magnetopause shadowing and subsequent rapid outward radial transport. Even in the case of limited compression of the magnetosphere, $\mathrm{Ni}$ et al. (2011) showed that there is a clear correlation between electron PSD dropouts and the solar wind pressure pulse, owing to a combination of magnetopause shadowing and outward radial diffusion. The latter can be achieved under the presence of ULF waves, a regularly occurring phenomenon, which can violate the third invariant condition and allow for electron radial diffusion (Mann et al., 2012).

In this work we attempt an assessment of selected mechanisms (substorm injections and wave-particle interactions via chorus and Pc5 waves) that contribute to the variability of the electron population in the outer radiation belt. To that end, we examine the PSD in phase space coordinates as well as wave activity (ULF Pc5 and whistler chorus) to compare and contrast the evolution of equatorial mirroring, relativistic and sub-relativistic electron population throughout Earth's outer radiation belt for two different geomagnetic storms: one storm resulting in an overall enhancement (17 March 2013) and the other resulting in depletion (12 September 2014) of the PSD throughout the outer belt.

\section{Data selection and methodology}

We use electron differential fluxes from the Magnetic Electron Ion Spectrometer (MagEIS) Medium M75 and High instruments (Blake et al., 2013), on board the Van Allen Probes. Fluxes are converted to PSD for fixed first and second adiabatic invariants for a range of values and $K<0.05 G^{1 / 2} R_{\mathrm{E}}$ (equatorial mirroring electrons with pitch angles $90 \pm \sim 15^{\circ}$ ) by applying the method described by Chen et al. $(2005,2007)$ using magnetic field measurements from the fluxgate magnetometers of Van Allen Probes (Kletzing et al., 2013). The use of PSD at fixed phase space coor- dinates (PSCs) allows us to both track particles and to identify regions and times when the adiabatic assumption breaks down (injection events, fast loss events), and therefore to automatically filter out the so-called $D_{\mathrm{ST}}$ effect. All values of the invariants $K$ and $L^{*}$ were calculated at each measurement point using the Tsyganenko and Sitnov (2005) magnetospheric field model (TS05). In addition, magnetic field measurements from the fluxgate magnetometers of RBSP (3 min resolution) as well as ground measurements obtained from SuperMAG collaboration were used to determine the average Pc5 power. A continuous wavelet transform with the Morlet wavelet as the basis function has been applied to analyze them in the time-frequency domain (Balasis et al., 2013). Prior to the time-frequency analysis using wavelet transforms, a high-pass Butterworth filter with a cut-off frequency of $0.9 \mathrm{mHz}$ was applied to obtain the wavelet power spectra covering the Pc5 frequency range (typically between 2 and $7 \mathrm{mHz}$ ). To quantify the temporal evolution of ULF wave activity, we calculated the weighted sum of the wavelet spectrum over Pc5 wave frequencies. The ULF wave-derived diffusion coefficients are then obtained from the aforementioned ground and in situ measurements. Ozeke et al. (2012) have demonstrated that the electric term is dominant over the magnetic one, over a wide range of parameters. Nevertheless, the magnetic term is more easily calculated from satellite data, since variations of $|B|$ correspond to compressional waves, and $|B|$ is easily measured; and relative changes in the value of $D_{\mathrm{LL}}^{B}$ are qualitatively, if not quantitatively, similar to changes in the value of $D_{\mathrm{LL}}^{E}$. Therefore, we calculate $D_{\mathrm{LL}}^{B}$ for indicative values of $\mu$, under the simplifying assumption that the whole ULF power falls in wave mode 1 . The reason that increasing the wave $m$ value does not significantly affect the magnetic field diffusion coefficient is because increasing $m$ also increases the resonant wave frequency and at higher wave frequencies the magnetic field PSD is much lower (Ozeke et al., 2012). On the other hand, we have obtained $D_{\mathrm{LL}}^{E}$ from the IMAGE (International Monitor for Auroral Geomagnetic Effects) (Tanskanen et al., 2009) and CARISMA (Canadian Array for Realtime Investigations of Magnetic Activity) (Mann et al., 2008) magnetometer array data using the mapping method of Ozeke et al. (2009). That method is restricted to daytime measurements and relies on ground stations that do not always match the location of the spacecraft in question. However, they are useful in providing a more accurate estimation of the total diffusion coefficient, whenever concurrent measurements are available.

Moreover we infer chorus wave amplitudes from the ratio of precipitating and trapped electron fluxes over the energy of $30-100 \mathrm{keV}$ (measured by POES satellites) applying the method described by $\mathrm{Li}$ et al. (2013). In this way we can estimate the chorus wave intensity in broad MLT coverage, which cannot be obtained from in situ chorus wave measurements by equatorial satellites alone. Supplementary measurements of $1 \mathrm{~min}$ averaged values of solar wind speed, pressure and interplanetary magnetic field (IMF) as well as 
geomagnetic indices SYM-H and AL from the NASA/OMNI database $^{1}$ are also considered.

\section{Detailed event analysis}

\subsection{Enhancement event}

An overview of the 16-18 March 2013 period of interest is shown in Fig. 1a-c. An interplanetary coronal mass ejection (ICME) on 17 March (arrival time at 05:00 UT) increased the solar wind speed from its quiet values to approximately $750 \mathrm{~km} \mathrm{~s}^{-1}$ and dynamic pressure to approximately $16 \mathrm{nPa}$ while the IMF magnitude reached $21 \mathrm{nT}$. Pressure gradually decreased to the pre-storm levels by midnight UT of the same day while speed gradually decreased by the end of the recovery phase on 18 March. The strongly southward IMF $(\approx 20 \mathrm{~h}$ duration $)$ associated with the ICME caused an intense storm (SYM-H index reached $-130 \mathrm{nT})$ and an interval of enhanced substorm activity (AL reached $-2100 \mathrm{nT}$ ) for the rest of day 17 until the beginning of the recovery phase. During 18 March there is no substorm activity or pressure enhancements. In Fig. 1d we show calculations of the magnetopause and plasmapause location using the models of Shue et al. (1998) and O'Brien and Moldwin (2003), respectively. Clearly the location of the magnetopause starts at a distance of $L \sim 11$ and reaches $L \sim 6$ during the main phase of the storm while it recovers to pre-storm distances afterward. The plasmapause exhibits a similar behavior; it starts at $L \sim 4$, while it moves to $L \sim 3$ during the main and recovery phase of the storm and then it recovers to slightly lower than the pre-event levels on 19 March.

Right after the storm sudden commencement (SSC) and the beginning of the substorm activity, the global chorus amplitudes increase by more than 1 order of magnitude for all L shells (Fig. 1e). This enhanced chorus activity lasts until the end of the main phase of the storm - which coincides with the end of the substorm activity - and decreases to the pre-storm levels during the recovery phase. This is consistent with the results of Meredith et al. $(2001,2002)$, who showed the dependence of chorus activity on substorm injections.

ULF wave activity, calculated from the magnetic field measurements of the Van Allen Probes in the nightside magnetosphere, shows similar behavior (Fig. 1f). There is an enhancement of Pc5 power up to 3 orders of magnitude right after the SSC that lasts until the beginning of the recovery phase of the storm. The subsequent decrease of Pc5 activity to the pre-storm levels coincides with the gradual decrement of dynamic pressure and solar wind speed. This is consistent with the theory of generation of ULF waves (Mann and Wright, 1999) due to instabilities (e.g., Kelvin-Helmholtz) at the flanks of the magnetosphere. Fälthammar (1965) and Elkington et al. (2003) proposed that fluctuations in the geomagnetic field produced by ULF waves may cause adiabatic

\footnotetext{
${ }^{1}$ http://omniweb.gsfc.nasa.gov/
}

radial diffusion of the radiation belt electrons. As a consequence the diffusion coefficients (Fig. 1g) are enhanced up to 3 orders of magnitude during the main phase of the storm for the whole outer belt $(3.5<L<6.5)$ and gradually drop to the pre-storm levels. The enhancement of radial diffusion is the same for the dayside magnetosphere as well (Fig. 1h-i); diffusion coefficients are enhanced up to 3 orders of magnitude during the main phase of the storm and then return to the pre-storm level values.

Time profiles of PSD for fixed adiabatic invariants are shown in Fig. 2. Each point is defined as the average PSD calculated from RBSP A and B after a binning in $L^{*}$ values. As shown, the overall net effect of the storm is enhancement of the electron population but there are slight differences depending on the electrons' energy. The population with $\mu=100 \mathrm{MeVG}^{-1}$ (panel a) increases right after the SSC. By the afternoon of 17 March, PSD has reached its maximum (up to 4 orders of magnitude) that coincides with the maximum of substorm activity. After the beginning of the recovery phase of the storm, PSD remains enhanced until the end of the storm. We note that there seems to be a dependence of the enhancement on the L shell; enhancement is more pronounced for lower L shells and, in addition, PSD is almost decreased to the pre-storm levels for $L=5.25$. The $300 \mathrm{MeV} \mathrm{G}^{-1}$ electron population (panel b) shows similar behavior except the enhancement is simultaneous for all $\mathrm{L}$ shells. The 600 and $900 \mathrm{MeV} \mathrm{G}^{-1}$ population show different behavior. Right after the SSC there is a PSD depletion (up to an order of magnitude) that coincides with the maximum compression of the magnetopause, which is the result of magnetopause shadowing (Boyd et al., 2014). After the depletion there is a sudden enhancement of PSD that reaches its maximum value at the end of the main phase of the storm (a few hours later than the lower $\mu$ population). Again, PSD remains enhanced until the end of the storm. We note that the dependence of the enhancement on $\mathrm{L}$ shell is not apparent in the high $\mu$ population (as $\mu$ increases the enhancement is more or less of the same order of magnitude).

\subsection{Depletion event}

An overview of the 11-13 September 2014 period of interest is shown in Fig. 3a-c. There is a high-speed stream that caused $\mathrm{a} \approx 10 \mathrm{~h}(00: 00-10: 00 \mathrm{UT}$ on 12 September) increase of speed (up to $450 \mathrm{~km} \mathrm{~s}^{-1}$ ) and pressure (up to $10 \mathrm{nPa}$ ) as well as some sporadic substorm activity but no storm (the minimum SYM-H index reached $-20 \mathrm{nT}$ ). After a $3 \mathrm{~h}$ quiet period an ICME arrives at 15:00 UT of 12 September. Solar wind speed is increased from its quiet values to approximately $750 \mathrm{~km} \mathrm{~s}^{-1}$ and dynamic pressure to approximately $20 \mathrm{nPa}$ while the IMF magnitude reached $30 \mathrm{nT}$. The strongly southward IMF associated with the ICME caused a shortlived but intense storm (SYM-H index reached $-100 \mathrm{nT}$ ) and an approximately $2 \mathrm{~h}$ interval of enhanced substorm activity (AL reached $-1200 \mathrm{nT}$ ) at the end of day 12. Speed grad- 
ヨ)

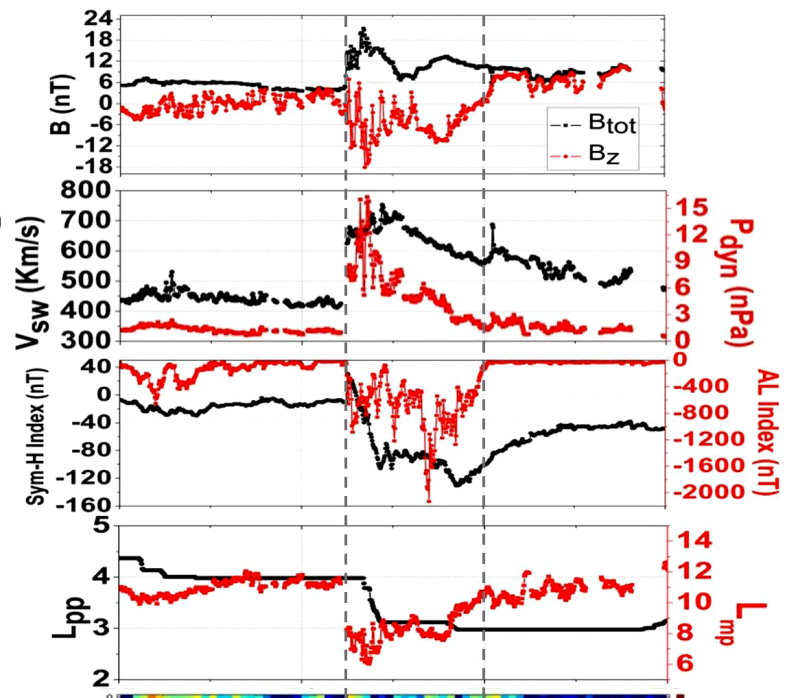

e)

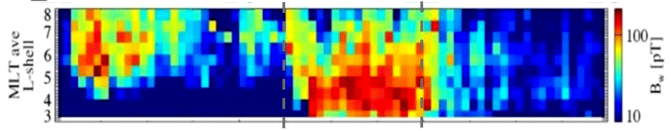

f)

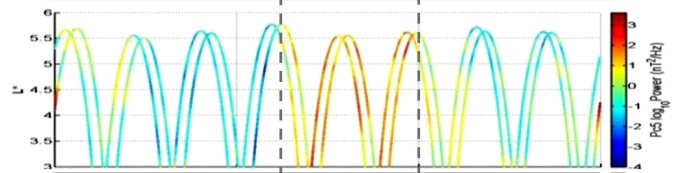

g)

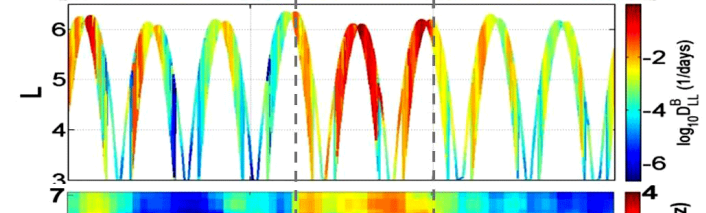

h)

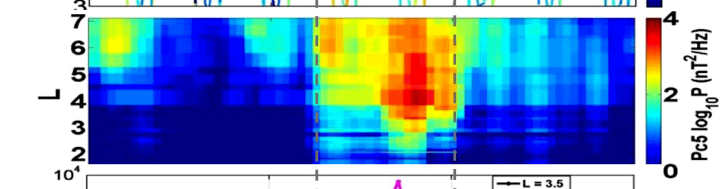

i)

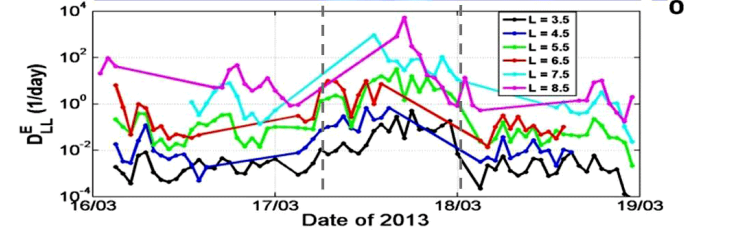

Figure 1. (a-d) Values of solar wind parameters and geomagnetic indices with $1 \mathrm{~min}$ resolution, during the time period $16-$ 18 March 2013. Top to bottom: average IMF and its $z$ component $\left(B_{z}\right)$, solar wind speed and dynamic pressure, geomagnetic indices SYM-H and AL, models of magnetopause (Shue et al., 1998) and plasmapause (O'Brien and Moldwin, 2003) location. (e) Chorus wave intensity averaged over all MLTs based on the POES electron measurements. (f) Pc5 power from RBSP for the nightside magnetosphere $(22: 00<\mathrm{MLT}<04: 00)$. (g) Averaged values of $D_{\mathrm{LL}}^{B}$ corresponding to RBSP magnetic field measurements at the nightside magnetosphere $(22: 00<\mathrm{MLT}<04: 00)$. (h) Pc5 power for the dayside magnetosphere obtained from ground observations (06:00 $<\mathrm{MLT}<11: 00)$. (i) Averaged values of $D_{\mathrm{LL}}^{E}$ at the dayside magnetosphere $(06: 00<M L T<11: 00)$ obtained from the CARISMA and IMAGE ground magnetometer arrays. The vertical dashed lines circumscribe the main phase of the storm.
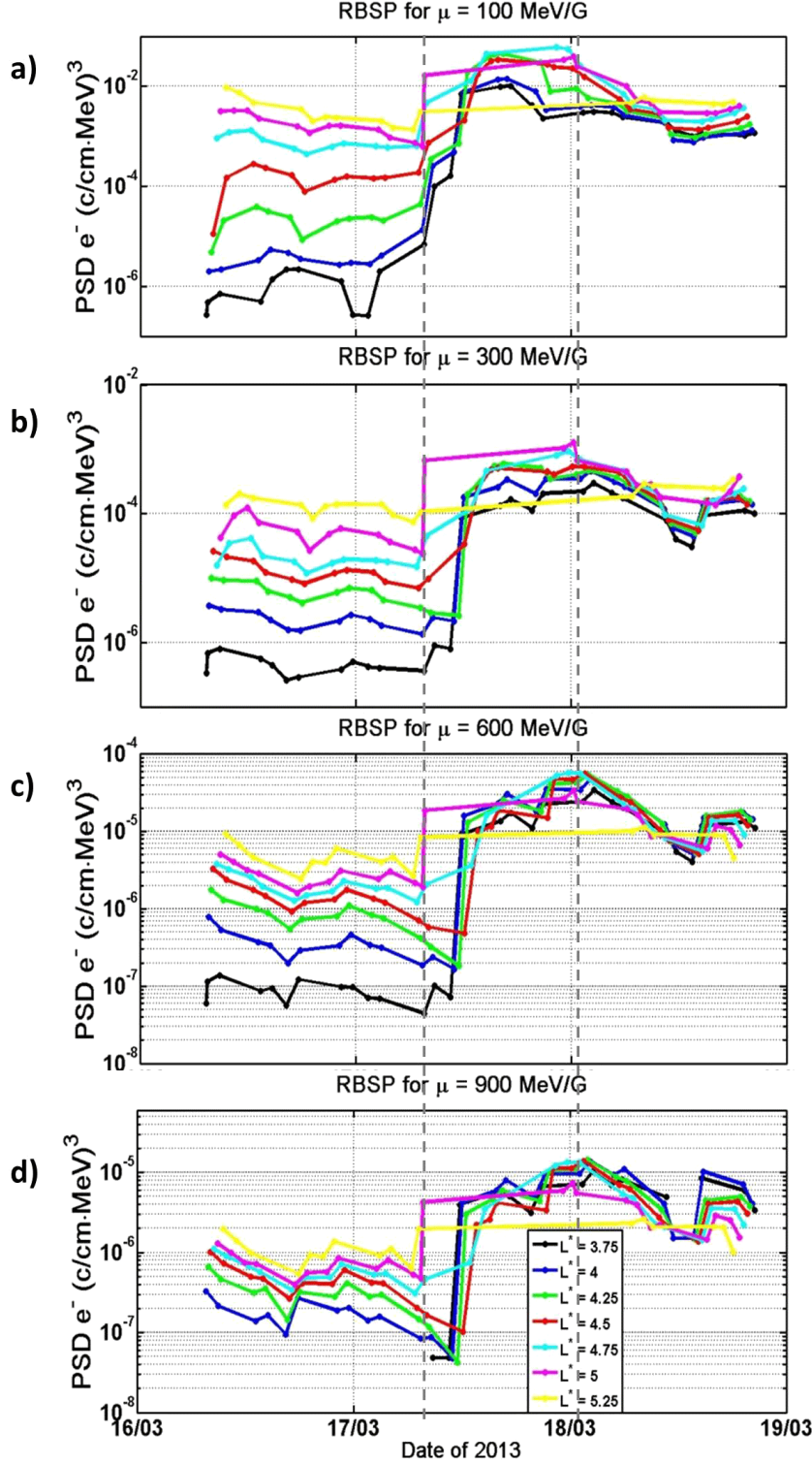

Figure 2. Nightside PSD time profiles obtained from RBSP flux measurements for fixed adiabatic invariants during the 16-18 March time period. Each panel corresponds to different value of $\mu$ (100, 300,600 and $900 \mathrm{MeV} \mathrm{G}^{-1}$ respectively) and each color curve to different value of $L^{*}$. The vertical dashed lines circumscribe the main phase of the storm.

ually decreases on 13 September but never reaches the prestorm levels. In addition, strong fluctuations of pressure are present during the whole recovery phase of the storm, yet there is no sign of substorm activity. The location of the magnetopause (panel d) starts at a distance of $L \sim 10$ and reaches $L \sim 6$ during the main phase of the storm while it recovers to larger than the pre-storm distances afterwards. The plasmapause exhibits a similar behavior; it starts at $L \sim 4$, while it moves to $L \sim 3$ during the main phase of the storm and then it recovers to pre-event levels on 14 September. 
The global chorus amplitudes are increased less than 1 order of magnitude (Fig. 3e), and the enhancement is very limited both in space $(3.5<L<5.5)$ and time (only during the minimum SYM-H). This enhancement coincides with the strong but also limited in time (a couple of hours) substorm activity indicating once again the dependence of chorus intensity on substorm injections.

ULF wave activity in the nightside magnetosphere shows completely different behavior (Fig. 3f). There is an enhancement of Pc5 power caused by a high-speed stream during the beginning of 12 September but Pc5 recover to the previous levels. With the arrival of the ICME in the afternoon, we observe an enhancement of more than 3 orders of magnitude (at all $\mathrm{L}$ shells). This enhancement is weakened during the storm recovery phase (up to 2 orders of magnitude); still, Pc5 power remains high, compared to the pre-storm levels, even at the end of the storm. This coincides with the fact that both solar wind speed and pressure are still enhanced during the recovery phase of the storm. As a consequence the diffusion coefficients (Fig. 3g) are enhanced up to 3 orders of magnitude during the main phase of the storm while the enhancement persists for $L>4$ during the recovery phase. The enhancement of radial diffusion is almost the same for the dayside magnetosphere (Fig. 3h-i); diffusion coefficients are enhanced up to 3 orders of magnitude during the main phase of the storm and then show sporadic and weak enhancements above the pre-storm levels.

Time profiles of PSD for fixed adiabatic invariants are shown in Fig. 4. The overall net effect of this storm is completely different, depending on the electrons' energy. The population with $\mu=100 \mathrm{MeV} \mathrm{G}^{-1}$ (panel a) increases right after the SSC and before midnight of 13 September; PSD for high $\mathrm{L}$ shells has reached its maximum that coincides with the maximum of substorm activity. After the beginning of the recovery phase of the storm, PSD for $L^{*}<4.5$ remains enhanced until the end of the storm while PSD for $L^{*}>4.5$ recovers to the pre-storm levels. We note that the dependence of the PSD enhancement on the L shell appears in both events. As we move to higher values of $\mu$ the behavior of the electron population is completely different. The $300 \mathrm{MeV} \mathrm{G}^{-1}$ electron population (panel b) shows an enhancement during the maximum chorus activity (main phase of the storm) and a depletion for $L^{*}>4.5$ right before the beginning of the recovery phase of the storm. By the end of the recovery phase, the electron population at high $\mathrm{L}$ shells has returned to the pre-storm levels while the low $\mathrm{L}$ shell population shows slight enhancement. For the higher $\mu$ electron population the aforementioned depletion is more abrupt and deep (especially for higher $\mathrm{L}$ shells) and the net effect of the storm is depletion and very slow recovery of PSD that exceeds the time interval of this study. a)

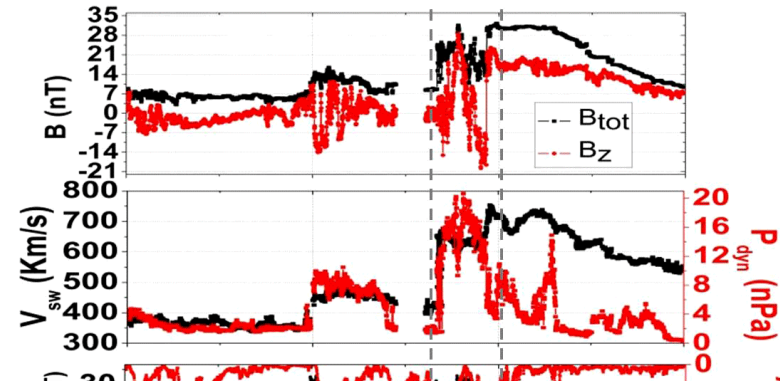

c)

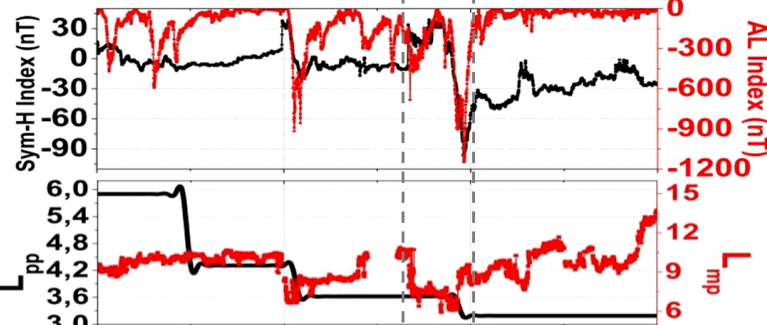

e)

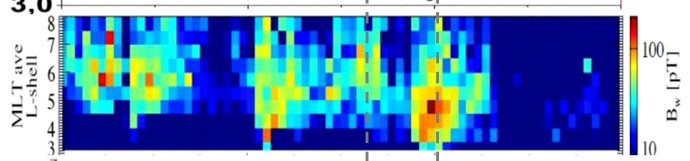

f)

g)

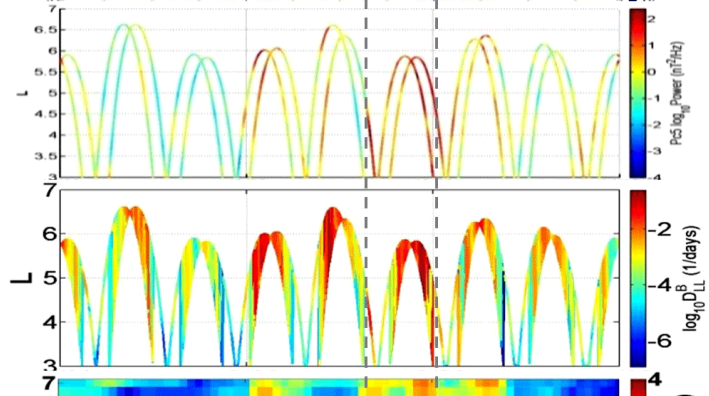

h)

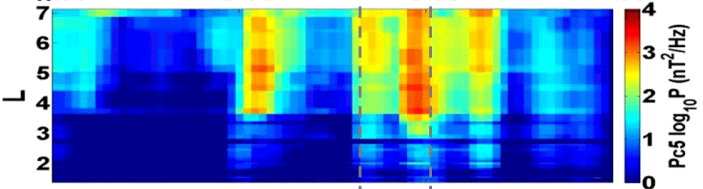

i)

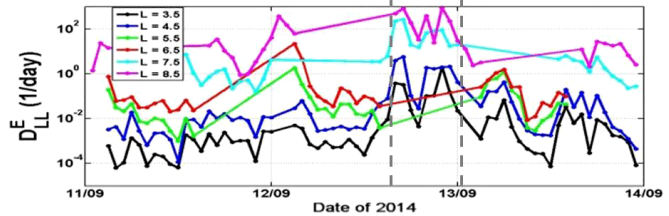

Figure 3. Same as Fig. 1 during the time period 11-13 September 2014.

\section{Discussion}

In order to study the contribution of different mechanisms to the outer radiation belt dynamics we chose to investigate the impact of ICMEs. The reason for that is that ICMEs drive a variety of magnetospheric processes that are relevant for radiation belt dynamics. They have a high solar wind speed and much larger ULF wave power than their surroundings. There are also indications that fluctuating IMF and $P_{\mathrm{dyn}}$ that are associated with ICMEs can enhance substorm occurrence and thus chorus wave activity (Hietala et al., 2014). 

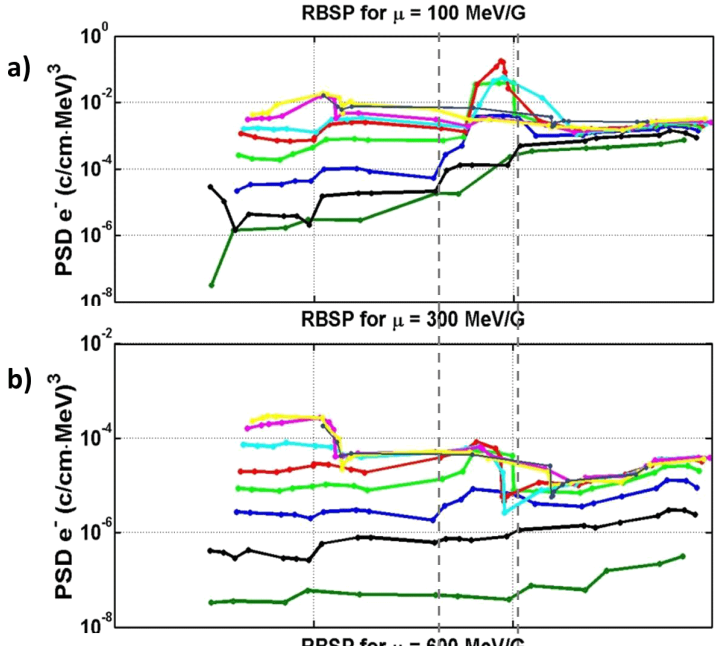

c)

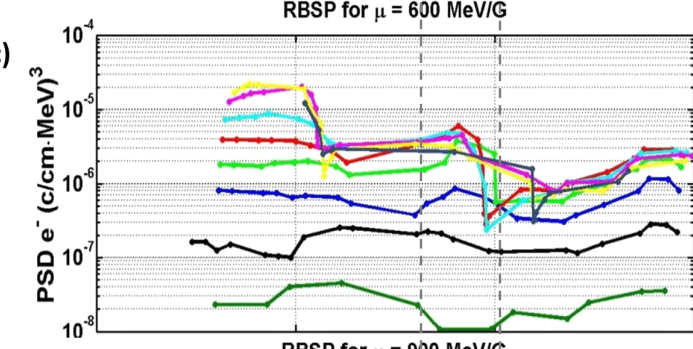

d)

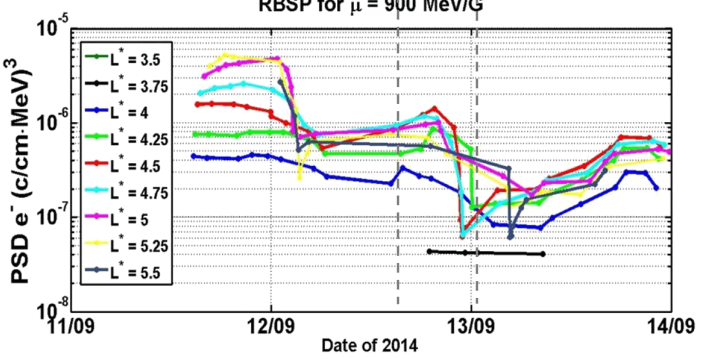

Figure 4. Same as Fig. 2 for the 11-13 September 2014 time period.

Both examined events exhibited intense storms (as indicated by the SYM-H index) caused by the arrival of an ICME. The pressure pulse was high enough to compress the magnetosphere to low $\mathrm{L}$ shells in both cases, but there are pronounced differences that connect the solar windmagnetic field-waves-electron population chain of events. During the March 2013 storm, the $z$ component of the IMF was continuously negative for more than $18 \mathrm{~h}$ and produced an equivalent time interval of intense substorm activity while speed and pressure gradually and smoothly decreased to the pre-storm levels. On the other hand, during the September 2014 storm the strong southward component of the IMF lasted for approximately $4 \mathrm{~h}$ and produced an equivalent interval of substorm activity while speed and pressure showed intense fluctuations during the recovery phase of the storm. During both recovery phases no substorm activity was present.
During the first storm, pronounced wave activity (both chorus and Pc5) at all $\mathrm{L}$ shells during the main phase was exhibited while there was no activity at all during the recovery phase. The latter coincides with the absence of substorm activity as well as the absence of fluctuations and the gradual decrement of speed and pressure on 18 March. The second storm exhibited very limited (in time and space) chorus activity, due to the short-lived main phase and substorm activity, but long-lasting Pc5 activity due to the continuously enhanced and fluctuating solar wind speed and pressure. The latter is consistent with the statistical study of Liu et al. (2010), who showed, over a 21-month period, that ULF wave activity (in the range of Pc4-5) is strongly correlated with solar wind speed and pressure in $4-9 R_{\mathrm{E}}$.

The net effect of the two storms was completely different and moreover dependent on the energy of the electrons. The March 2013 event suggests that the intense series of substorms and the associated generation of chorus waves are the mechanisms that lead to enhancement of the electron population. This is consistent with the results of Li et al. (2014) and Turner et al. (2014). In detail, the low $\mu$ electron population was enhanced right after the beginning of the substorm activity while a few hours later higher energy electrons reached the maximum values of PSD. This result (amongst others in the literature) is a verification of the scenario proposed by Horne et al. (2005) in which substorm- and shock-related injections are responsible for the access of energetic electrons (approximately hundreds of $\mathrm{keV}$ ) to the outer belt and the generation of chorus activity. These seed electrons are accelerated by chorus waves to even higher (relativistic) energies. We note that the electron acceleration occurs even though the Pc5 activity was pronounced (and comparable to the chorus activity) and outward diffusion, as indicated by the diffusion coefficient calculations, was present. Moreover, electron PSDs remain enhanced even during the storm recovery phase. This coincides with the absence of significant activity both in the solar wind (speed, pressure and IMF) and inside the magnetosphere (AL index and waves).

On the other hand, observations of the September 2014 event suggest that the outward diffusion driven by the pronounced and long-lasting Pc5 activity was, possibly, the dominant mechanism for $>300 \mathrm{MeV} \mathrm{G}^{-1}$ electron PSD depletion. In detail, the low $\mu$ electron population was enhanced right after the beginning of the short-lived substorm activity due to the shock/substorm-related injections. Furthermore, the intense substorm injection (during the main phase of the storm) drove chorus wave activity which, consequently, was also short-lived. Nevertheless, despite their short duration, chorus waves efficiently accelerated at $4.25<L<5$ to relativistic energies $\left(\mu>300 \mathrm{MeV} \mathrm{G}^{-1}\right)$. During the recovery phase of the storm, the $>300 \mathrm{MeV} \mathrm{G}^{-1}$ electron population is depleted. This depletion coincides with the absence of significant chorus wave activity and the enhanced Pc5 power. We note that, as explained below, outward diffusion seems to be the only mechanism (combined with mag- 
netopause shadowing or not) able to cause such a pronounced depletion of the $>300 \mathrm{MeV} \mathrm{G}^{-1}$ electron population.

Alternative loss mechanisms could be the scattering of the $>300 \mathrm{MeV} \mathrm{G}^{-1}$ electron population into the loss cone via wave-particle interactions due to plasmaspheric hiss or EMIC waves. However, Usanova et al. (2014) have shown that EMIC waves are able to affect only ultra-relativistic electrons at higher latitudes but not the equatorial mirroring population examined here. Moreover, the activity of plasmaspheric hiss is limited mostly inside the plasmapause (Thorne et al., 2005), which was compressed up to $L=3$ during both of the events. Even if the plasmapause were located at higher L shells, hiss-driven electron precipitation has a timescale from $\approx 1$ day to tens of days depending on energy (Meredith et al., 2007; Thorne et al., 2013; Jaynes et al., 2014) and not a few hours. In addition, this depletion is consistent with the results of Loto'aniu et al. (2010), who showed that non-adiabatic losses on 25 June 2008 event occurred over a timescale of $1-4 \mathrm{~h}$.

\section{Conclusions}

In this work we attempt an assessment of selected mechanisms that contribute to the variability of the electron population in the outer radiation belt. To that end, we examine the PSD in phase space coordinates as well as wave activity (ULF Pc5 and whistler chorus) to compare and contrast the evolution of equatorially mirroring, relativistic and subrelativistic electron population throughout Earth's outer radiation belt for two different geomagnetic storms: (a) one storm that exhibited pronounced chorus and Pc5 wave activity for the same time interval and resulted in an overall enhancement of the electron population (16-18 March 2013) and (b) another that exhibited short-lived chorus wave activity but pronounced and long-lasting Pc5 wave activity which resulted in depletion of the $>300 \mathrm{MeV} \mathrm{G}^{-1}$ electron population (11-13 September 2014) throughout the outer belt. All of this evidence leads us to the following conclusions:

1. There is a $300 \mathrm{MeV} \mathrm{G}^{-1}$ threshold in $\mu$ that separates not only the source of relativistic electron population inside the outer belt after the arrival of a prominent pressure pulse but also the mechanisms that contribute to its variability. Electrons below this limit are accelerated due to substorm/shock injections regardless of the net effect of various mechanisms on higher $\mu$ electrons while electrons above this threshold are accelerated locally by interactions with chorus waves.

2. Concerning the $>300 \mathrm{MeV} \mathrm{G}^{-1}$ electrons, the comparison of the two events shows that during similar intervals of pronounced chorus and Pc5 wave activity, the relative effect is higher for chorus waves (i.e., the chorus-driven acceleration exceeds the Pc5-driven losses).
3. Finally, it is the long-lasting Pc5 power enhancements (with absence of chorus wave activity) that lead to PSD depletion of the $>300 \mathrm{MeV} \mathrm{G}^{-1}$ electron population via outward diffusion.

A future statistical study with a large-enough number of events is necessary in order to verify the aforementioned results.

Acknowledgements. This research has been co-financed by the European Union (European Social Fund - ESF) and Greek national funds through the Operational Program Education and Lifelong Learning of the National Strategic Reference Framework (NSRF) Research Funding Program: Thales. Investing in knowledge society through the European Social Fund. We are thankful to the THEMIS and RBSP-ECT teams, NASA's OMNI and NGDC for online data access and data analysis tools. RBSP-ECT data and analysis software are made available at the http://www.rbsp-ect.lanl.gov through funding by JHU/APL contract no. 967399 under NASA's Prime contract no. NAS5-01072. The analysis at UCLA was supported by the NASA grants NNX11AD75G, NNX11AR64G, NNX13AI61G, NNX14AI18G, and NNX15AF61G, and the Air Force Young Investigator program.

The topical editor B. Mauk thanks J. Bortnik and one anonymous referee for help in evaluating this paper.

\section{References}

Baker, D. N. and Daglis, I. A.: Radiation belts and ring current, in Space Weather - Physics and Effects, edited by: Bothmer, V. and Daglis, I. A., 173-202, Springer Verlag, Berlin, 2007.

Balasis, G., Daglis, I. A., Georgiou, M., Papadimitriou, C., and Haagmans, R.: Magnetospheric ULF wave studies in the frame of Swarm mission: A time-frequency analysis tool for automated detection of pulsations in magnetic and electric field observations, Earth Planets Space, 65, 1385-1398, doi:10.5047/eps.2013.10.003, 2013.

Blake, J. B., Carranza, P. A., Claudepierre, S. G., Clemmons, J. H., Crain Jr., W. R., Dotan, Y., Fennell, J. F., Fuentes, F. H., Galvan, R. M., George, J. S., Henderson, M. G., Lalic, M., Lin, A. Y., Looper, M. D., Mabry, D. J., Mazur, J. E., McCarthy, B., Nguyen, C. Q., O’Brien, T. P., Perez, M. A., Redding, M. T., Roeder, J. L., Salvaggio, D. J., Sorensen, G. A., Spence, H. E., Yi, S., and Zakrzewski, M. P.: The Magnetic Electron Ion Spectrometer (MagEIS) Instruments Aboard the Radiation Belt Storm Probes (RBSP) Spacecraft, Space Sci. Rev., 179, 383-421, doi:10.1007/s11214-013-9991-8, 2013.

Bortnik, J., Cutler, J. W., Dunson, C., and Bleier, T. E.: An automatic wave detection algorithm applied to Pc1 pulsations, J. Geophys. Res., 112, A04204, doi:10.1029/2006JA011900, 2007.

Boyd, A. J., Spence, H. E., Claudepierre, S. G., Fennell, J. F., Blake, J. B., Baker, D. N., Reeves, G. D., and Turner, D. L.: Quantifying the radiation belt seed population in the 17 March 2013 electron acceleration event, Geophys. Res. Lett., 41, doi:10.1002/2014GL059626, 2014.

Chen, Y., Friedel, R. H. W., Reeves, G. D., Onsager, T. G., and Thomsen, M. F.:Multisatellite determination of the relativistic 
electron phase space density at geosynchronous orbit: Methodology and results during geomagnetically quiet times, J. Geophys. Res., 110, A10210, doi:10.1029/2004JA010895, 2005.

Chen, Y., Friedel, R. H. W., Reeves, G. D., Cayton, T. E., and Christensen, R.: Multi-satellite determination of the relativistic electron phase space density at geosynchronous orbit: An integrated investigation during geomagnetic storm times, J. Geophys. Res., 112, A11214, doi:10.1029/2007JA012314, 2007.

Elkington, S. R., Hudson, M. K., and Chan, A. A.: Resonant acceleration and diffusion of outer zone electrons in an asymmetric geomagnetic field, J. Geophys. Res., 108, A1116, doi:10.1029/2001JA009202, 2003.

Fälthammar, C. G.: Effects of time-dependent electric fields on geomagnetically trapped radiation, J. Geophys. Res., 70, 2503-2516, doi:10.1029/JZ070i011p02503, 1965.

Hietala, H., Kilpua, E. K. J., Turner, D. L., and Angelopoulos, V.: Depleting effects of ICME-driven sheath regions on the outer electron radiation belt, Geophys. Res. Lett., 41, 2258-2265, doi:10.1002/2014GL059551, 2014.

Horne, R. B., Thorne, R. M., Glauert, S. A., Albert, J. M., Meredith, N. P., and Anderson, R. R.: Timescale for radiation belt electron acceleration by whistler mode chorus waves, J. Geophys. Res., 110, A03225, doi:10.1029/2004JA010811, 2005.

Jaynes, A. N., Li, X., Schiller, Q. G., Blum, L. W., Tu, W., Turner, D. L., Ni, B., Bortnik, J., Baker, D. N., Kanekal, S. G., Blake, J. B., and Wygant, J.: Evolution of relativistic outer belt electrons during an extended quiescent period, J. Geophys. Res., 119, 9558-9566, doi:10.1002/2014JA020125, 2014.

Kim, K. C., Lee, D. Y., Kim, H. J., Lyons, L. R., Lee, E. S., Ozturk, M. K., and Choi, C. R.: Numerical calculations of relativistic electron drift loss effect, J. Geophys. Res., 113, A09212, doi:10.1029/2007JA013011, 2008.

Kim, K. C. and Lee, D. Y.: Magnetopause structure favorable for radiation belt electron loss, J. Geophys. Res., 119, 5495-5508, doi:10.1002/2014JA019880, 2014.

Kletzing, C. A., Kurth, W. S., Acuna, M., MacDowall, R. J., Torbert, R. B., Averkamp, T., Bodet, D., Bounds, S. R., Chutter, M., Connerney, J., Crawford, D., Dolan, J. S., Dvorsky, R., Hospodarsky, G. B., Howard, J., Jordanova, V., Johnson, R. A., Kirchner, D. L., Mokrzycki, B., Needell, G., Odom, J., Mark, D., Pfaff Jr., R., Phillips, J. R., Piker, C. W., Remington, S. L., Rowland, D., Santolik, O., Schnurr, R., Sheppard, D., Smith, C. W., Thorne, R. M., and Tyler, J.: The Electric and Magnetic Field Instrument Suite and Integrated Science (EMFISIS) on RBSP, Space Sci. Rev., 179, 127-181, doi:10.1007/s11214-013-9993-6, 2013.

Li, W., Ni, B., Thorne, R. M., Bortnik, J., Green, J. C., Kletzing, C. A., Kurth, W. S., and Hospodarsky, G. B.: Constructing the global distribution of chorus, wave intensity using measurements of electrons by the POES satellites and waves by the Van Allen Probes, Geophys. Res. Lett., 40, 4526-4532, doi:10.1002/grl.50920, 2013.

Li, W., Ni, B., Thorne, R. M., Ma, Q., Ni, B., Bortnik, J., Baker, D. N., Spence, H. E., Reeves, G. D., Kanekal, S. G., Green, J. C., Kletzing, C. A., Kurth, W. S., Hospodarsky, G. B., Blake, J. B., Fennell, J. F., and Claudepierre, S. G.: Radiation belt electron acceleration by chorus waves during the 17 March 2013 storm, J. Geophys. Res., 119, 4681-4693, doi:10.1002/2014JA019945, 2014
Li, X., Baker, D. N., O’Brien, T. P., Xie, L., and Zong, Q. G.: Correlation between the inner edge of outer radiation belt electrons and the innermost plasmapause location, Geophys. Res. Lett., 33, L14107, doi:10.1029/2006GL026294, 2006.

Liu, W., Sarris, T. E., Li, X., Ergun, R., Angelopoulos, V., Bonnell, J., and Glassmeier, K. H.: Solar wind influence on Pc4 and Pc5 ULF wave activity in the inner magnetosphere, J. Geophys. Res., 115, A12201, doi:10.1029/2010JA015299, 2010.

Loto'aniu, T. M., Singer, H. J., Waters, C. L., Angelopoulos, V., Mann, I. R., Elkington, S. R., and Bonnell, J. W.: Relativistic electron loss due to ultralow frequency waves and enhanced outward radial diffusion, J. Geophys. Res., 115, A12245, doi:10.1029/2010JA015755, 2010.

Mann, I. R. and Wright, A. N.: Diagnosing the excitation mechanisms of Pc5 magnetospheric flank waveguide modes and FLRs, Geophys. Res. Lett., 26, 2609-2612, doi:10.1029/1999GL900573, 1999.

Mann, I. R., Milling, D. K., Rae, I. J., Ozeke, L. G., Kale, A., Kale, Z. C., Murphy, K. R., Parent, A., Usanova, M., Pahud, D. M., Lee, E. A., Amalraj, V., Wallis, D. D., Angelopoulos, V., Glassmeier, K. H., Russell, C. T., Auster, H. U., and Singer, H. J.: The Upgraded CARISMA Magnetometer Array in the THEMIS Era, Space Sci. Rev., 141, 413-451, doi:10.1007/s11214-008-9457-6, 2008.

Mann, I. R., Murphy, K. R., Ozeke, L. G., Rae, I., Milling, D. K., and Kale, A.: The role of ultralow frequency waves in radiation belt dynamics, Geoph. Monog. Series, 199, 69-92, doi:10.1029/2012GM001349, 2012.

Meredith, N. P., Horne, R. B., and Anderson, R. R.: Substorm dependence of chorus amplitudes: Implications for the acceleration of electrons to relativistic energies, J. Geophys. Res., 106, 13165-13178, doi:10.1029/2000JA900156, 2001.

Meredith, N. P., Horne, R. B., Iles, R. H. A., Thorne, R. M., Heynderickx, D., and Anderson, R. R.: Outer zone relativistic electron acceleration associated with substormenhanced whistler mode chorus, J. Geophys. Res., 107, 1144, doi:10.1029/2001JA900146, 2002.

Meredith, N. P., Horne, R. B., Glauert, S. A., and Anderson, R. R.: Slot region electron loss timescales due to plasmaspheric hiss and lightning-generated whistlers, J. Geophys. Res., 112, A08214, doi:10.1029/2007JA012413, 2007.

Ni, B., Shprits, Y., Hartinger, M., Angelopoulos, V., Gu, X., and Larson, D.: Analysis of radiation belt energetic electron phase space density using THEMIS SST measurements: Cross-satellite calibration and a case study, J. Geophys. Res., 116, A03208, doi:10.1029/2010JA016104, 2011.

O'Brien, T. P. and Moldwin, M. B.: Empirical plasmapause models from magnetic indices, Geophys. Res. Lett., 30, 1152, doi:10.1029/2002GL016007, 2003.

O’Brien, T. P., Lorentzen, K. R., Mann, I. R., Meredith, N. P., Blake, J. B., Fennell, J. F., Looper, M. D., Milling, D. K., and Anderson, R. R.: Energization of relativistic electrons in the presence of ULF power and MeV microbursts: Evidence for dual ULF and VLF acceleration, J. Geophys. Res., 108, 1329, doi:10.1029/2002JA009784, 2003.

Ozeke, L. G., Mann, I. R., and Rae, I. J.: Mapping guided Alfvén wave magnetic field amplitudes observed on the ground to equatorial electric field amplitudes in space, J. Geophys. Res., 114, A01214, doi:10.1029/2008JA013041, 2009. 
Ozeke, L. G., Mann, I. R., Murphy, K. R., Rae, I. J., Milling, D. K., Elkington, S. R., Chan, A. A., and Singer, H. J.: ULF wave derived radiation belt radial diffusion coefficients, J. Geophys. Res., 117, A04222, doi:10.1029/2011JA017463, 2012.

Reeves, G. D., McAdams, K. L., Friedel, R. H. W., and O'Brien, T. P.: Acceleration and loss of relativistic electrons during geomagnetic storms, Geophys. Res. Lett., 30, 1529, doi:10.1029/2002GL016513, 2003.

Schulz, M. and Lanzerotti, L. J.: Particle diffusion in the radiation belts, Physics and Chemistry in Space, Berlin, Springer, Germany, 81-85, 1974.

Shprits, Y. Y., Thorne, R. M., Friedel, R., Reeves, G. D., Fennell, J., Baker, D. N., and Kanekal, S. G.: Outward radial diffusion driven by losses at magnetopause, J. Geophys. Res., 111, A11214, doi:10.1029/2006JA011657, 2006.

Shprits, Y. Y., Meredith, N. P., and Thorne, R. M.: Parameterization of radiation belt electron loss timescales due to interactions with chorus waves, Geophys. Res. Lett., 34, L11110, doi:10.1029/2006GL029050, 2007.

Shue, J. H., Song, P., Russell, C. T., Steinberg, J. T., Chao, J. K., Zastenker, G., Vaisberg, O. L., Kokubun, S., Singer, H. J., Detman, T. R., and Kawano, H.: Magnetopause location under extreme solar wind conditions, J. Geophys. Res., 103, 1769117700, doi:0148-0227/98/98JA-01103, 1998.

Tanskanen, E. I.: A comprehensive high-throughput analysis of substorms observed by IMAGE magnetometer network: Years 1993-2003 examined, J. Geophys. Res., 109, A05220, doi:10.1029/2003JA010294, 2009.

Thorne, R. M., Horne, R. B., Glauert, S., Meredith, N. P., Shprits, Y. Y., Summers, D., and Anderson, R. R.: The Influence of WaveParticle Interactions on Relativistic Electron Dynamics During Storms, in: Inner Magnetosphere Interactions, New Perspectives From Imaging, edited by: Burch, J., Schulz, M., and Spence, H., AGU, Washington, DC, USA, 159, 101 pp., 2005.
Thorne, R. M., Horne, R. B., Glauert, S., Meredith, N. P., Shprits, Y. Y., Summers, D., and Anderson, R. R.: Evolution and slow decay of an unusual narrow ring of relativistic electrons near $\mathrm{L} \sim 3.2$ following the September 2012 magnetic storm, Geophys. Res. Lett., 114, 3507-3511, doi:10.1002/grl.50627, 2013.

Turner, D. L., Shprits, Y., Hartinger, M., and Angelopoulos, V.: Explaining sudden losses of outer radiation belt electrons during geomagnetic storms, Nature Physics Lett., 8, 208-212, doi:10.1038/NPHYS2185, 2012.

Turner, D. L., Angelopoulos, V., Li, W., Hartinger, M. D., Usanova, M., Mann, I. R., Bortnik, J., and Shprits, Y.: On the storm-time evolution of relativistic electron phase space density in Earth's outer radiation belt, J. Geophys. Res., 118, 2196-2212, doi:10.1002/jgra.50151, 2013.

Turner, D. L., Angelopoulos, V., Li, W., Bortnik, J., Ni, B., Ma, Q., Thorne, R. M., Morley, S. K., Henderson, M. G., Reeves, G. D., Usanova, M., Mann, I. R., Claudepierre, S. G., Blake, J. B., Baker, D. N., Huang, C. L., Spence, H., Kurth, W., Kletzing, C., and Rodriguez, J. V.: Competing source and loss mechanisms due to wave-particle interactions in Earth's outer radiation belt during the 30 September to 3 October 2012 geomagnetic storm, J. Geophys. Res., 119, 1960-1979, doi:10.1002/2014JA019770, 2014.

Tsyganenko, N. A. and Sitnov, M. I.: Modeling the dynamics of the inner magnetosphere during strong geomagnetic storms, J. Geophys. Res., 110, A03208, doi:10.1029/2004JA010798, 2005.

Usanova M. E., Drozdov, A., Orlova, K., Mann, I. R., Shprits, Y., Robertson, M. T., Turner, D. L., Milling, D. K., Kale, A., Baker, D. N., Thaller, S. A., Reeves, G. D., Spence, H. E., Kletzing, C., and Wygant, J.: Effect of EMIC waves on relativistic and ultrarelativistic electron populations: Groundbased and Van Allen Probes observations, Geophys. Res. Lett., 41, 1375-1381, doi:10.1002/2013GL059024, 2014. 